\title{
New Perspectives on Enteric Pathogenic Bacteria Bacterium Escherichia coli
}

\section{Abdulaziz Radhi S Aljohni ${ }^{1 *}$, Tawfeek Rezeek Almotiri², Tariq Ibrahim Alhazmy $^{3}$, Ghazi Khalil Suliman Alqouzi ${ }^{4}$ and Fahd Omar AL Mhmodi ${ }^{5}$}

${ }^{1}$ Phd Microbiology, Department of Laboratory at King Fahad Hospital, Medina, Saudi Arabia

${ }^{2}$ MSc in Molecular Pathology, Department of Laboratory at King Fahad Hospital, Medina, Saudi Arabia

${ }^{3}$ MSc Senior Specialist, Laboratory Histopathology Department at Forensic Medicine Centre, Madina, Saudi Arabia

${ }^{4}$ MSc Laboratory Histopathology at Al-Qunfudhah Health Regional Laboratory, Saudi

Arabia

${ }^{5}$ Medical Laboratory Technology, Al-Qunfudhah Health Regional Laboratory, Saudi

Arabia

*Corresponding Author: Abdulaziz Radhi S Aljohni, Phd Microbiology, Department of Laboratory at King Fahad Hospital, Medina, Saudi Arabia.
Received: November 12, 2021

Published: November 29, 2021

(C) All rights are reserved by Abdulaziz Radhi S Aljohni., et al.

\section{Abstract}

E. coli is a bacteria present in the guts of humans and warm-blooded animals. Most E. coli strains are safe. Toxigenic E. coli (STEC), for example, may cause severe foodborne illness. Humans acquire it via contaminated meals including raw or undercooked ground beef, raw milk, and raw vegetables and sprouts.

STEC generates Shiga-toxins, named after Shigella dysenteriae toxins. STEC grows well in temperatures between 7 to $50^{\circ} \mathrm{C}$, with an optimum of $37^{\circ} \mathrm{C}$. Some STEC may grow in acidic meals with pH as low as 4.4 and water activity (aW) as low as 0.95 .

STEC is eradicated by thoroughly heating meals to $70^{\circ} \mathrm{C}$ or above. The most common STEC serotype is E. coli 0157:H7, however other serotypes have been implicated in occasional cases and outbreaks.

Keywords: Escherichia coli; Commonly Found Humans; Warm-blooded Animals; Taxonomy; Symptoms; Treatment

\section{Introduction}

In 1982, two outbreaks of a particularly bloody diarrheal illness led to the discovery of Escherichia coli 0157:H7 [1]. Since then, epidemiologic, clinical, and laboratory studies have significantly extended our understanding of this bacterium and the disorders it causes. Other enterohemorrhagic E. coli serotypes eventually shared this pathogenic potential, and the group was named enterohemorrhagic E. [2]. The most researched member of this group is E. coli 0157:H7. This organism's infections are becoming more common, owing to growing interest in the organism and commercial reagents for identification, as well as increased frequency and geographic reach. The disease's epidemics would have drawn attention, and the bacterium was originally detected using standard

Citation: Abdulaziz Radhi S Aljohni., et al. "New Perspectives on Enteric Pathogenic Bacteria Bacterium Escherichia coli". Acta Scientific Microbiology 4.12 (2021): 111-118. 
microbiologic procedures. The HUS was originally characterized in 1955 [3]. So E. coli 0157:H7 and its illnesses are relatively new. This organism is a model for enterohemorrhagic E. coli and other developing bacterial diseases. The presence of E. coli 0157:H7 in the feces of hemorrhagic colitis patients, its absence in healthy stools, and the pathogenicity of the bacterium and its toxins in animal models all meet the three Koch-Henle criteria for causation [4]. The presence of the organism in implicated food vehicles and animal sources, as well as particular antibody responses in infected people, bolster these claims. Because of the severity of the illness and lack of effective medication, much of our information comes from epidemiologic field research and clinical observation. Infection microbiologic, clinical and toxin biochemistry have all been well reported in excellent reviews [8]. This review will summarize E. coli epidemiology.

\section{Taxonomy}

Proteobacteria Phylum, Gammaproteobacteria Class, Enterobacterales Order, Enterobacteriaceae Family Escherichia genus.

- $\quad$ Escherichia coli Leclerc 1962

- $\quad$ Leclercia adecarboxylata was assigned to the genus Leclercia.

- $\quad$ Tamura., et al. 1987

- $\quad$ Huys., et al. 2003 - Escherichia albertii.

Burgess., et al. 1973 transferred Escherichia blattae to the genus Shimwellia blattae Priest and Barker Escherichia coli (Bacterium coli commune, Escherich 1885), Castellani and Chalmers 1919, Escherichia coli (Bacterium coli commune, Escherich 1885), Escherichia coli (Bacterium coli commune, Escherich 1885), Escherichia coli (Bacterium coli commune- Escherichia fergusonii Farmer., et al. 1985.

- $\quad$ Escherichia hermannii Brenner., et al. 1983

- $\quad$ Escherichia marmotae Lių., et al. 2015

- $\quad$ Escherichia ruysiae van der Putten., et al. 2021

- $\quad$ Escherichia vulneris Brenner., et al. 1983 - moved to genus Pseudescherichia [9].

\section{Symptoms}

Symptoms of E. coli 0157 infections normally develop 3 to 4 days after the individual has been exposed to the bacterium. Symp- toms, on the other hand, might occur as early as 24 hours after the injury or as late as one week afterward.

Some of these symptoms include abdominal pain or severe abdominal cramping, often beginning suddenly watery diarrhea, beginning a few hours after the pain begins bright red bloody stools around a day later, resulting from the toxin's damage to the intestines nausea and, in some cases, vomiting fever, usually below 101 degrees Fahrenheit fatigue, resulting from dehydration and the loss of fluids and electrolytes in some cases

Some persons may not show any signs of illness, yet they are still capable of spreading the virus to others.

\section{Complications}

- The majority of individuals recover completely within a week.

- However, around $10 \%$ of the population is at danger of getting hemolytic uremic syndrome, according to Trusted Source (HUS). The majority of those there are little children and the elderly.

- Hemolysis, or the breaking apart of red blood cells, is a characteristic of HUS. Anemia, a low platelet count, and renal failure might result as a result of this.

- In the kidneys, platelets, which are the blood cells responsible for blood clotting, clump together and restrict blood flow, leading in ischemia, which is a condition marked by diminished blood flow.

- If this is not addressed, it might result in renal failure. Reduced platelet counts increase the likelihood of experiencing bleeding difficulties.

- $\quad$ Patients who have these clots may also have difficulties with their central nervous system (CNS), which may include problems with their brain and spinal cord.

- $\quad$ Seizures, paralysis, brain swelling, and coma are all possible consequences. This disease is deadly in around 3 to 5 percent of patients.

- When it comes to newborns and young children, HUS is the most common cause of acute renal failure. 
- $\quad$ HUS commonly manifests itself 5 to 8 days following the commencement of the diarrheal symptoms. It is a medical emergency that necessitates in-patient hospitalization.

Risk factors

- $\quad$ Some individuals are more susceptible to E. coli infections.

- Immunocompromised patients are more prone to problems. This includes AIDS patients, immunosuppressive drug users, and chemotherapy patients.

- Infections are more likely to occur in patients who have had stomach surgery or who use alkaline medications.

- $\quad$ Serious infections and consequences affect young children and the elderly.

\section{Sources and transmission}

Most STEC information focuses on serotype 0157:H7, which is readily distinguished biochemically from other $E$. coli strains. This pathogen's reservoir seems to be cattle. The virus has also been identified in other ruminants (such sheep and goats) and birds (like chickens and turkeys).

Humans get E. coli 0157:H7 via contaminated foods including raw or undercooked ground beef and raw milk. Cross-contamination during food preparation (with beef and other meat products, infected surfaces and kitchen utensils) may also cause illness. Undercooked hamburgers, dry cured salami, unpasteurized freshpressed apple cider, yogurt, and raw milk cheese have all been linked to E. coli 0157:H7 outbreaks.

Increasingly, outbreaks are linked to the intake of fruits and vegetables (particularly sprouts, spinach, lettuce, coleslaw, and salad), which may have been contaminated by animal feces during production or processing. STEC has been identified in ponds, streams, wells, and water troughs, and may live for months in manure and water-trough sediments. Both polluted drinking water and recreational waterways have been recorded.

Person-to-person interaction is vital in oral-fecal transmission. Asymptomatic carriers display no clinical symptoms yet may infect others. Adults excrete STEC in a week or less, while youngsters might take longer. Visiting farms and other places where the pub- lic may come into touch with farm animals is also a risk factor for STEC infection.

\section{Prevention}

Control measures are required at all stages of the food chain, from agricultural production on the farm through processing, manufacturing, and preparation of meals in both commercial enterprises and private kitchens, to prevent illness from spreading.

\section{Industry}

Multiple mitigation measures for ground beef might potentially lower the frequency of disease outbreaks in this animal product (for example, screening the animals pre-slaughter to reduce the introduction of large numbers of pathogens in the slaughtering environment). Though proper slaughtering methods help to limit the contamination of carcasses by excrement, they do not ensure that STEC will not be present in the final product.

The education of farmworkers, slaughterhouse employees, and others engaged in food production on the proper sanitary handling of foods is vital to minimize microbial contamination to a bare minimum. To effectively eliminate STEC from foods, a bactericidal treatment such as heating (for example, cooking or pasteurization), irradiation, or other means must be used.

\section{Household}

E. coli 0157:H7 infection is treated with antibiotics like other foodborne diseases. Basic food hygiene measures, like those specified in the WHO's "Five keys to safer food," may prevent the spread of germs that cause many foodborne infections, such as Salmonella enterica.

Safer food supply requires five keys:

- $\quad$ Keep the place tidy.

- $\quad$ Sort raw from cooked.

- $\quad$ Cook for a long time.

- $\quad$ Mend the food storage temperatures.

- Use non-hazardous water and raw materials.

All of these recommendations, especially "cook thoroughly" until the center reaches $70^{\circ} \mathrm{C}$, should be followed in every case. 
Wash fruits and vegetables well before eating, especially if eaten fresh. Vegetables and fruits should be peeled if possible. Vulnerable populations should avoid raw or undercooked animal products, raw milk, and raw milk products (such as young children and the elderly).

Because the germs may be spread from person to person, food to person, and animal to animal, regular hand washing is suggested. Particularly for caregivers of small children, the elderly, or immunocompromised patients.

A variety of STEC infections have been associated with recreational water exposure. As a consequence, protecting such aquatic bodies and drinking water sources from animal waste is vital [4].

\section{Producers of fruits and vegetables}

The "Five keys to producing safer fruits and vegetables," developed by the World Health Organization, educates rural workers on how to reduce microbial contamination of fresh produce during planting, growing, harvesting, and storage.

The following are the five keys to more secure fruit and vegetable growth:

- $\quad$ Keep your hygiene in check.

- Prevent contamination from animal feces.

- Make use of feces that have been treated.

- $\quad$ Evaluate and manage the risks associated with irrigation water.

- Harvesting and storage equipment that is clean and dry.

\section{Treatment}

These infections will go away on their own. Due to a lack of laboratory diagnosis, symptomatic infections are typically treated empirically. It differs by region. In most cases, EAEC strains are resistant to fluoroquinolones, azithromycin/clavulanic acid, and nalidixic acid [12]. In the feces of Polish children with diarrhea, Sobieszczanska and colleagues [13] discovered EAEC microbes resistant to ampicillin, tetracycline, trimethoprim, sulfamethoxazole, and chloramphenicol. EAEC bacteria in Thailand were resistant to the antibiotics co-trimoxazole and amoxicillin, which are often used to treat gastroenteritis. Fluoroquinolones were shown to be effective against the majority of strains [14]. Three clinical investigations on EAEC diarrheal illness have been conducted, one on HIV-infected patients in developing countries and two on travelers to impoverished countries. Glandt and colleagues compared the clinical effects of ciprofloxacin with a placebo in 29 US visitors with EAEC diarrhea. For three days, 16 passengers were given ciprofloxacin $500 \mathrm{mg}$ daily, whereas 13 were given a placebo. The ciprofloxacin patients had much less diarrhea than the controls ( 35 vs 56 hours). 43 people with EAEC diarrhea were compared to placebo in a multicenter study including US travelers visiting Guatemala, Kenya, and Guadalajara, Mexico. Thirty individuals were given rifaximin (200 or $400 \mathrm{mg}$ daily for three days) and thirteen were given a placebo. Patients who took rifaximin had a shorter illness than those who took a placebo (22 vs 72 hours). In a crossover trial of 24 HIV-positive people with EAEC diarrhea, those who were given ciprofloxacin (500 mg twice daily for 7 days) had $50 \%$ fewer bowel movements and $42 \%$ fewer other enteric symptoms than those who were given the placebo. 52 These three studies recommend fluoroquinolones, particularly ciprofloxacin $500 \mathrm{mg}$ bid for 3-7 days and rifaximin 200 or $400 \mathrm{mg}$ bid for 3 days, to treat symptomatic EAEC infections [14].

\section{Epidemiology}

Enteric E. coli is found in the natural flora of many animals. Human infections are caused by consuming tainted food (undercooked meat or diseased fresh produce such as salad greens), drinking tainted water (animal or human waste), or practicing poor hygiene. Enteric $E$. coli infections are difficult to measure since the factors that induce diarrhea are often unknown. These infections seem to be substantial causes of infantile diarrhea in the developing world, although they are mild and self-limiting in the developed world. EHEC, EAEC, and STEAEC pathotypes are found in the majority of food poisoning incidents in rich nations.

Many hundred million instances of diarrhea and several tens of thousands of fatalities are recorded each year in children under the age of five in poor nations, with ETEC being the most often identified bacterial enteropathogen in these children. ETEC is a bacterium that is a member of the ETEC family. ETEC, the most common cause of travelers' diarrhea, affects anywhere from 10 percent to 60 percent of individuals who get ill, depending on where they go and how sick they are $[15,16]$. ETEC may be responsible for a total of 10 million cases of travelers' diarrhea every year, based on 
the extrapolation of these figures. Among the animals infected with ETEC include cattle, neonatal and post-weaning pigs, among other types of animals. The acquisition of colonization factors (CF) rather than the creation of animal-specific lineages are responsible for the acquisition of host specificity [17].

In addition to ETEC, EAEC is the second most prevalent cause of travelers' diarrhea, and its presence in both endemic and epidemic illnesses is becoming more well recognized in recent years. This enteric pathogen has been suggested as a significant enteric pathogen affecting AIDS patients [18], and it causes chronic diarrhea in children in underdeveloped countries. There has been no description of an animal reservoir for EAEC, indicating that it is no longer present in the human population. The 2011 E. coli foodborne epidemic in Germany was caused by an EAEC strain (0104:H4) that has acquired typical EHEC traits, most notably the production of Stx, as well as other EHEC characteristics. There were 852 cases of HUS, with 32 deaths, and 3469 cases of non-HUS STEAEC, with 18 deaths. Patients infected with STEAEC 0104:H4 developed hemolytic uremic syndrome (HUS) at a high rate, with a mortality rate of 1 percent; there were 852 cases of HUS, with 32 deaths, and 3469 cases of non-HUS STEAEC, with 18 deaths. Taken along with the recent large-scale epidemic and the preceding outbreaks of Stx2positive 0104:H4 [19], STEAEC might now be regarded as a new pathotype of enteric E. coli, according to the authors. To confirm STEAEC as an emergent pathotype, it will be necessary to continue to identify this unique population of hybrid EAEC/EHEC strains throughout time.

The significance of DAEC in the pathogenesis of enteric illness is still debated. However, concerns with the cross-reactivity of one of the typical detection probes raise doubts regarding the significance of DAEC in diarrheagenic illness in children, according to some research. A link between DAEC and illness has been shown in particular age demographics (children aged 18 mo-5 y or 13-24 mo) [20-22], however further epidemiological research is needed if DAEC is to be recognized as a unique intestinal $E$. coli pathotype in the future.

Whether the relationship between AIEC and Crohn's disease (CD) is causal or symptomatic is still up in the air. A combination of the two is most probable, with a genetic predisposition to developing CD aggravated by microbial infection (including AIEC) becom- ing active $\mathrm{CD}$ as a result of the combination. Several AIEC strains have been identified as being related with CD lesions in ileal, neoterminal ileal, and colonic tissues, as well as in animal models [23]. The presence of an enhanced immune response to E. coli in CD patients implies that E. coli may be involved in the pathogenesis of the disease [24].

In the developed world, epidemiological data for enteric E. coli infections is generally collected based on toxin production rather than pathotypes or serotypes, and infections are therefore commonly referred to as Stx-producing E. coli (STEC) or Verotoxigenic E. coli (VEC). In the developing world, epidemiological data for enteric $E$. coli infections is generally collected based on toxin production rather than pathotypes or serotypes, and infections (VTEC). These classifications may encompass the whole of a pathotype (for example, all EHEC strains are STECs) or just a portion of a pathotype (for example, all STAEC strains are STECs), and they can be further subdivided into STEC/VTEC 0157, which refers to the most frequent EHEC serogroup. Foodborne infections cause an estimated 9.4 million hospitalizations and 1,351 fatalities in the United States each year, according to estimates from 2011. However, even though STEC 0157 infections accounted for only 4\% of laboratory-confirmed foodborne infections in the United States between 1996 and 2005, STEC 0157 infections had the highest case fatality rate across the population and the highest annual population mortality rate in children aged 0-4 years. According to data from the European Union for 2009, VTEC was responsible for 1 percent of laboratory-confirmed zoonotic infections, with 7 percent of individuals infected having the hemolytic uremic syndrome. As a result, although STEC/VTEC infection rates are relatively modest when compared to Campylobacter and Salmonella infection rates, the severity of the sickness and high case fatality rates make these diseases a serious public health issue $[25,26]$.

As a result, although EPEC was the first E. coli strain to be widely recognized as a cause of diarrheagenic epidemics in the developed world, its prevalence has dropped, and EPEC outbreaks are now uncommon in the developed world. In the developing world, however, it continues to be a significant cause of infant diarrhea, with recent estimates of EPEC prevalence among children with diarrhea ranging from 6-54 percent, even though high carriage rates among healthy controls make determining the contribution of EPEC to disease difficult. Persistent diarrhea has been seen in patients with 
atypical EPEC (that is, those that do not carry the EAF plasmid that encodes bundle-forming pili (BFP)) [28].

Even though EIEC and Shigella may be separated by simple biochemical tests, the two pathogens share the same virulence mechanisms and illness signs in most cases. Strains of EIEC and Shigella seem to have developed separately but have several traits in common [29] and the EIEC strains that are now in use may simply be intermediates between E. coli and Shigella in origin. To learn more about this pathotype, we recommend that you read the great review on the Shigella species that are included in this edition of the journal [30].

\section{Detection and diagnosis}

Given the need for selective enrichment, one of the most pressing questions is how to identify Shiga toxin-producing bacteria in either the feces of sick patients or contaminated food, which is a major worry. Several strategies for routine diagnosis have previously been outlined in detail. 139 The examination of the cytotoxicity of bacterial culture supernatants to eukaryotic cells, however, continues to be the gold standard for Stx identification. A multiplex PCR assay that incorporates the stx gene, as well as other virulence genes, might be beneficial in screening for STEC in bacterial confluent growth zones or in colonies of fermenting and non-fermenting bacteria obtained from SMAC, for example [30].

To diagnose STEC, a large number of tests have been developed based on the detection of Stx1 and/or Stx2, which represent the key virulence factors of this E. coli subtype. The sensitivity and specificity of a test are dependent on the test format and the manufacturer of the instrument [32]. Nonetheless, the standards by which each manufacturer assesses its tests vary, and as a result, a direct comparison of the performance characteristics of different immunoassays has not been carried up to this point. Furthermore, many commercially accessible diagnostics are out of reach for underdeveloped nations due to their high cost. This has been shown in earlier studies using a variety of immunoassay formats, such as a combination of rabbit anti-Stx 1 and -2 sera by indirect ELISA, or polyclonal and monoclonal antibodies in a capture ELISA test for the detection of STEC, to name a few examples [33].

The standardized procedures are repeatable, rapid, and simple to execute, and they demonstrate great sensitivity in identifying Stx by capturing ELISA, even in isolates with low levels of production.
There has been no evaluation of these assays for industrial quality control or commercial availability, although the projected cost of the assay is under US\$70 per 96 detections, making it a reasonably affordable option for impoverished nations. As a consequence of the rebuilding of these monoclonal antibodies, single-chain fragment variable ( $\mathrm{scFv}$ ) fragments were produced. It was discovered that Stx2-scFv could be obtained from a bacteria-induced culture and had diagnostic ability; the scFv fragment was able to recognize the majority of Stx2-producing strains with 77.3 percent sensitivity (confidence interval: 60.3 to 92 percent) and no reactivity was observed with the non-producing strains, indicating specificity as high as 100 percent (confidence interval: 86.8-100 percent) [34]. It is worth noting that none of the currently available immunoenzymatic tests for the detection of Stx $1 / 2$ toxin use recombinant antibodies generated in bacteria, which will undoubtedly lower the prices of the diagnostic assays [35].

\section{Conclusion}

A newly developed real-time PCR assay for the detection of $E$. coli, E. coli 0157, and the Shiga-like toxin genes Stx1 and Stx 2 can be used as a quick test to reliably type $E$. coli colonies grown on culture plates. The assay was developed to detect E. coli, E. coli 0157, and the Shiga-like toxin genes Stx 1 and Stx2. When bacteria were first concentrated in m-TSB broth, culture-PCR techniques were used to identify the presence of culturable E. coli, E. coli 0157 , and Stx gene-containing bacteria. Cultivation-PCR tests were very sensitive, specific, and simple to execute, and they enabled the identification of the E. coli 0157 and Stx genes in the presence of large quantities of bacteria from the environment (wastewater). CulturePCR techniques were effectively used to surface and wastewater samples to identify E. coli and STEC 0157 in measurable quantities. When used in MPN format, the culture-PCR produced E. coli concentrations that were equivalent to those obtained using standard culture techniques.

\section{Bibliography}

1. Riley LW., et al. "Hemorrhagic colitis associated with a rare Escherichia coli serotype". The New England Journal of Medicine 308 (1983): 681-685.

2. Levine MM and Edelman R. "Enteropathogenic Escherichia coli of classic serotypes associated with infant diarrhea: epidemiology and pathogenesis". Epidemiologic Reviews 6 (1984): 31-51. 
3. Gasser C., et al. "Hamolytischuramische syndrome: Bilaterale nierenrindennekrosen bei akuten erworbenen hamolytischen anamien". Schweizerische Medizinische Wochenschrift 85 (1955): 205-9.

4. Evans AS. "Epidemiologic concepts". In: Evans AS, Brachman PS, eds. Bacterial infections of humans: epidemiology and control. 2nd ed. New York: Plenum Medical Book Company (1991): 3-57.

5. Riley LW. "The epidemiologic, clinical, and microbiologic features of hemorrhagic colitis". Annual Review of Microbiology 41 (1987): 383-407.

6. Karmali MA. "Infection by verocytotoxin producing Escherichia coli”. Clinical Microbiology Reviews 2 (1989): 15-38.

7. Griffin PM., et al. "Illnesses associated with Escherichia coli 0157:H7 infections". A broad Clinical Spectrum Annals of Internal Medicine 109 (1988): 705-712.

8. O'Brien AD and Holmes RK. "Shiga-like toxins". Microbiology and Molecular Biology Reviews 51.2 (1987): 206-220.

9. JG Holt., et al. "Facultatively Gram-Negative Rods". Subgroup 1. Family Enterobacteriaceae. Determinative Bacteriology, 9thedition, Williams, and Wilkins (1994): 175-189.

10. Huang DB., et al. "Enteroaggregative Escherichia coli: an emerging enteric pathogen". The American Journal of Gastroenterology 2010 (2010): 254159.

11. Glandt M., et al. "Enteroaggregative Escherichia coli as a cause of traveler's diarrhea: clinical response to ciprofloxacin". Clinical Infectious Diseases 29 (1999): 335-338.

12. Sobieszczanska B., et al. "Susceptibility to antimicrobial agents of enteroaggregative Escherichia coli strains isolated from children with diarrhea". Epidemiologic Reviews 57 (2003): 499-503.

13. Yamamoto T., et al. "Drug resistance and adherence to human intestines of enteroaggregative Escherichia coli". The Journal of Infectious Diseases 165 (1992): 744-749.
14. Infante RM., et al. "Enteroaggregative Escherichia coli diarrhea in travelers: response to rifaximin therapy". Clinical Gastroenterology and Hepatology 2 (2004): 135-138.

15. Nagy B and Fekete PZ. "Enterotoxigenic Escherichia coli in veterinary medicine". International Journal of Medical Microbiology 295 (2005): 443-454.

16. Sarantuya J., et al. "Typical enteroaggregative Escherichia coli is the most prevalent pathotype among E. coli strains causing diarrhea in Mongolian children". Journal of Clinical Microbiology 42 (2004): 133-139.

17. Dutta S., et al. "Use of PCR to identify enteroaggregative Escherichia coli as an important cause of acute diarrhoea among children living in Calcutta, India". Journal of Medical Microbiology 48 (1999): 1011-1016.

18. Wanke CA., et al. "Enteroaggregative Escherichia coli as a potential cause of diarrheal disease in adults infected with human immunodeficiency virus". The Journal of Infectious Diseases 178 (1998): 185-190.

19. Bielaszewska M., et al. "Characterisation of the Escherichia coli strain associated with an outbreak of haemolytic uraemic syndrome in Germany, 2011: a microbiological study". The Lancet Infectious Diseases 11 (2011): 671-676.

20. RKI. EHEC/HUS 0104:H4 -The outbreak is over. Robert Koch Institute (2011).

21. Mellmann A., et al. "Analysis of collection of hemolytic uremic syndrome-associated enterohemorrhagic Escherichia coli". Emerging Infectious Diseases 200814 (2008): 1287-1290.

22. Bae WK., et al. "A case of hemolytic uremic syndrome caused by Escherichia coli 0104:H4". Yonsei Medical Journal 47 (2006): 437.

23. Snelling AM., et al. "The commonly-used DNA probe for diffusely-adherent Escherichia coli cross-reacts with a subset of enteroaggregative E. coli". BMC Microbiology 9 (2009): 269. 
24. Gunzburg ST., et al. "Diffuse and enteroaggregative patterns of adherence of enteric Escherichia coli isolated from aboriginal children from the Kimberley region of Western Australia". The Journal of Infectious Diseases 167 (1993): 755-758.

25. Scaletsky IC., et al. "Diffusely adherent Escherichia coli as a cause of acute diarrhea in young children in Northeast Brazil: a case-control study". Journal of Clinical Microbiology 40 (2002): 645-648.

26. Darfeuille-Michaud A., et al. "High prevalence of adherent-invasive Escherichia coli associated with ileal mucosa in Crohn's disease". Gastroenterology 127 (2004): 412-421.

27. Rolhion N and Darfeuille-Michaud A. "Adherent-invasive Escherichia coli in inflammatory bowel disease". Inflammatory Bowel Diseases 13 (2007): 1277-1283.

28. Scallan E., et al. "Foodborne illness acquired in the United States”. Emerging Infectious Diseases 17 (2011): 1339-1340.

29. Barton Behravesh C., et al. FoodNet Working Group. "Deaths associated with bacterial pathogens transmitted commonly through food: foodborne diseases active surveillance network (FoodNet), 1996-2005". The Journal of Infectious Diseases 204 (2011): 263-267.

30. European Food Safety Authority. "The European Union Summary Report on Trends and Sources of Zoonoses, Zoonotic Agents and Food-borne Outbreaks in 2009". EFSA Journal 9 (2011): 2090-2468.

31. Gärtner JF and Schmidt MA. "Comparative analysis of locus of enterocyte effacement pathogenicity islands of atypical enteropathogenic Escherichia coli". Infection and Immunity 72.11 (2004): 6722-6728.

32. Ingle DJ., et al. "Evolution of atypical enteropathogenic E. coli by repeated acquisition of LEE pathogenicity island variants". Nature Microbiology 1 (2016): 15010.

33. Rocha LB., et al. "Interaction between Shiga toxin and monoclonal antibodies: binding characteristics and in vitro neutralizing abilities". Toxins 4 (2012): 729-747.
34. Luz D., et al. "Development and characterization of recombinant antibody fragments that recognize and neutralize in vitro stx2 toxin from Shiga toxin-producing Escherichia coli". PLoS One 10.3 (2015): 0120481.

35. L Hannah Gould., et al. Center of Disease Control of United States, Centers for Disease Control and Prevention. "Recommendations for diagnosis of Shiga toxin-producing Escherichia coli infections by clinical laboratories". Morbidity and Mortality Weekly Report 58 (2009): 1-12.

\section{Volume 4 Issue 12 December 2021}

(C) All rights are reserved by Abdulaziz Radhi S Aljohni., et al. 